Research Article

\title{
The Effectiveness of Scutellaria baicalensis on Migraine: Implications from Clinical Use and Experimental Proof
}

\author{
Chung-Chih Liao $\mathbb{D}^{1},{ }^{1}$ Ke-Ru Liao $\mathbb{D}^{2},{ }^{2}$ Cheng-Li Lin $\mathbb{D}^{3,4}$ and Jung-Miao Li ${ }^{10,5}$ \\ ${ }^{1}$ Graduate Institute of Chinese Medicine, College of Chinese Medicine, China Medical University, Taichung 40402, Taiwan \\ ${ }^{2}$ Department of Neurology, Yuanlin Christian Hospital, Yuanlin 51052, Taiwan \\ ${ }^{3}$ Management Office for Health Data, China Medical University Hospital, Taichung 40447, Taiwan \\ ${ }^{4}$ College of Medicine, China Medical University, Taichung 40402, Taiwan \\ ${ }^{5}$ Department of Chinese Medicine, Show Chwan Memorial Hospital, Changhua 50008, Taiwan \\ Correspondence should be addressed to Jung-Miao Li; rung-miau@hotmail.com
}

Received 24 July 2020; Revised 23 December 2020; Accepted 26 December 2020; Published 6 January 2021

Academic Editor: Arielle Cristina Arena

Copyright ( 2021 Chung-Chih Liao et al. This is an open access article distributed under the Creative Commons Attribution License, which permits unrestricted use, distribution, and reproduction in any medium, provided the original work is properly cited.

\begin{abstract}
Background. Scutellaria baicalensis (SB), a traditional Chinese medicine, is commonly used for the treatment of inflammatory and painful conditions. The purpose of the present study was to examine the effects of SB on migraine. Materials and Methods. We examined the clinical applications of SB based on the data obtained from Taiwan's National Health Insurance Research Database and confirmed that it was frequently used in Taiwan for the treatment of headaches. An experimental migraine model was established in rats by an intraperitoneal injection of nitroglycerin (NTG, $10 \mathrm{mg} / \mathrm{kg}$ ). Pretreatment with SB was given orally $30 \mathrm{~min}$ before NTG administration. The rats were subjected to migraine-related behaviour tests that were video-recorded and analysed using EthoVision XT 12.0 software. Results. The frequency of exploratory and locomotor behaviour was comparatively lower in the NTG group than that in the control group, while the frequency of resting and grooming behaviour increased. These phenomena were ameliorated by pretreatment with $1.0 \mathrm{~g} / \mathrm{kg} \mathrm{SB}$. The total time spent on the smooth surface was longer in the NTG group than that in the control group, but the time was shortened by pretreatment with $1.0 \mathrm{~g} / \mathrm{kg} \mathrm{SB}$. Conclusions. Pretreatment with $1.0 \mathrm{~g} / \mathrm{kg} \mathrm{SB}$ relieved migraine-related behaviours in the experimental NTG-induced migraine model. The outcome therefore demonstrated that pretreatment with $1.0 \mathrm{~g} / \mathrm{kg} \mathrm{SB}$ is beneficial for migraine treatment.
\end{abstract}

\section{Introduction}

Migraine is a prevalent and complex neurological disorder, characterized by recurrent unilateral, pulsating, moderateto-severe pain, aggravated by routine physical activity, and associated with nausea, photophobia, or phonophobia [1]. According to large-scale recent research, migraine affects approximately $12 \%$ of the population worldwide and has a higher prevalence among the female population, school/ college students, and urban residents $[2,3]$.

Traditional Chinese medicine (TCM) has been used for the treatment of migraine for thousands of years by using TCM theory experience. Recently, a large body of basic and clinical research confirmed the scientific benefit of TCM for the treatment of migraine [4-7]. The root of Scutellaria baicalensis (SB, known in Chinese medicine as Huang Qin) is a TCM herb, distributed in countries such as China, Japan, North Korea, Russia, and Mongolia [8]. It is known, from the experience of traditional Chinese doctors, to have analgesic, anti-inflammatory, and neuroprotective effects $[9,10]$. Migraine is now known as an inflammatory neurovascular disorder [11]. Although SB has anti-inflammatory and neuroprotective potency, clinical or experimental research on SB in TCM academia for the treatment of migraine has hardly been reported.

As a result, the present study first explored the clinical use of SB in Taiwan. Systemic administration of nitroglycerin (NTG) in rodents to induce hyperalgesia or migraine- 
related behaviours is one of the most widely accepted experimental migraine models [12-15]. Thereafter, we studied the effects of SB on migraine by behavioural analysis of an NTG-induced migraine rat model.

\section{Materials and Methods}

2.1. Clinical Applications of SB. The National Health Insurance program in Taiwan, which is an integration of all public insurance systems, offers insurance for conventional Western medicine and TCM $[16,17]$. Detailed information about TCM utilization was collected from the National Health Insurance Research Database (NHIRD).

We conducted an analysis of Longitudinal Health Insurance Database 2000 (LHID 2000), which is a random sample of one million enrollees from the NHIRD's longitudinal data spanning the period of 1997 to 2013. Diagnostic codes were collected from the International Classification of Diseases, Ninth Revision, Clinical Modification (ICD-9$\mathrm{CM}$ ). We extracted the top ten most common indications for SB from the primary diagnosis codes. This study was approved by the Institutional Review Board of China Medical University in central Taiwan (CMUH-104-REC2-115-R3).

2.2. Animals. Male Sprague Dawley rats, purchased from BioLASCO (Taipei, Taiwan), weighing 225-300 g were used in this study. A light-dark cycle of 12:12 h, relative humidity of $55 \% \pm 5 \%$, and room temperature of $23^{\circ} \mathrm{C} \pm 1{ }^{\circ} \mathrm{C}$ were maintained. Food and tap water were provided ad libitum. Animal use was approved by the Institutional Animal Care and Use Committee of Show Chwan Memorial Hospital (no. 106021) and followed the Guide for the Use of Laboratory Animals (National Academy Press).

2.3. SB Preparation. SB extract subtle granules were produced by Ko Da Pharmaceutical Co., Ltd. (Taoyuan, Taiwan). Scutellariae radix, the dried root of Scutellaria baicalensis Georgi (Fam. Labiatae), was purchased from Gansu province, People's Republic of China (Figure 1(a)). The origin and voucher specimens were identified and kept by Ko Da Pharmaceutical Co., Ltd. In brief, $210 \mathrm{~kg}$ of Scutellariae radix was extracted in 10 -fold $(\mathrm{w} / \mathrm{v})$ boiled water for $1 \mathrm{~h}$ followed by filtration through a 40 -mesh sieve. The filtrates were collected and subjected to vacuum concentration to produce $70 \mathrm{~kg}$ of extracts. The excipients, including $40.6 \mathrm{~kg}$ of starch, $28 \mathrm{~kg}$ of Scutellariae radix powder, and $1.4 \mathrm{~kg}$ of sodium carboxymethyl cellulose, were dried in a granulator, and then the extracts were added followed by granulation. The ratio of extracts and starch in SB extract subtle granules was $1: 1(\mathrm{w} / \mathrm{w})$.

Analytical high-performance liquid chromatography was performed using a Hitachi D-7000 interface equipped with an L-7100 pump, L-7455 detector, and L-7200 autosampler (Tokyo, Japan) to examine the baicalin content in the SB extract subtle granules. The test solution was prepared by mixing approximately $0.5 \mathrm{~g}$ of SB extract subtle granules with $30 \mathrm{~mL}$ mixture of acetonitrile and diluted phosphoric acid in a ratio of 28 and $72(\mathrm{v} / \mathrm{v})$ under heating reflux for
$30 \mathrm{~min}$. After centrifugation at $4000 \mathrm{rpm}$ for $10 \mathrm{~min}$, the supernatant was collected and added to a mixture of acetonitrile and diluted phosphoric acid at a ratio of 28 to $72(\mathrm{v} /$ v) to make up a final volume of $100 \mathrm{~mL}$ and then passed through a $0.45 \mu \mathrm{m}$ filter. Around $5 \mathrm{mg}$ of baicalin, with purity higher than $98 \%$ as claimed by the supplier (ChemFaces, Hubei, China), was mixed with $10 \mathrm{~mL}$ to obtain a stock standard solution, and $2 \mathrm{~mL}$ of stock standard solution was added to the mixture of acetonitrile and diluted phosphoric acid at a ratio of 28 to $72(\mathrm{v} / \mathrm{v})$ for a final volume of $20 \mathrm{~mL}$ to obtain the working standard solution. Chromatographic separation was carried out on a Mightysil RP18 column $(250 \times 4.6 \mathrm{~mm}, 5 \mu \mathrm{m})$ using an isocratic solvent system comprising a mixture of acetonitrile and diluted phosphoric acid at a ratio of 28 to $72(\mathrm{v} / \mathrm{v})$. The ultraviolet wavelength, flow rate, injection volume, and stop time were set at $270 \mathrm{~nm}, 1.0 \mathrm{~mL} / \mathrm{min}, 10 \mu \mathrm{L}$, and $30 \mathrm{~min}$, respectively. The content of baicalin in the SB extract subtle granules was $148.51 \mathrm{mg} / \mathrm{g}$ (Figure 1(b)).

2.4. Grouping. To induce migraine attacks, the rats were given an intraperitoneal (i.p.) injection of NTG $(10 \mathrm{mg} / \mathrm{kg})$ [14]. A total of 24 rats were randomly allocated into four groups $(n=6)$ as follows:

(1) Control group: i.p. injection of normal saline only

(2) NTG group: i.p. injection of NTG only

(3) Placebo group: oral administration of $1.0 \mathrm{~g} / \mathrm{kg}$ starch 30 min before i.p. injection of NTG

(4) SB-1.0 group: oral administration of $1.0 \mathrm{~g} / \mathrm{kg} \mathrm{SB}$ 30 min before i.p. injection of NTG

The dose of SB used in the present study was calculated based on the report by Nair and Jacob [18]. They reported that rat equivalent dose $(\mathrm{mg} / \mathrm{g})=$ human dose $(\mathrm{mg} / \mathrm{g}) \times 6.2$. We considered that an average human weighs $60 \mathrm{~kg}$. Therefore, based on the clinical human dosage of $9.6 \mathrm{~g}$, which is the rational dose commonly used in clinical TCM practice and suggested by pharmaceutical companies in Taiwan, we calculated the appropriate SB dosage for rats to be $1.0 \mathrm{~g} / \mathrm{kg}$.

The experimental procedure is shown in Figure 2.

\subsection{Rat Behaviour Tests}

2.5.1. Assessment of Spontaneous Nociceptive Behaviour. Thirty minutes after i.p. injection with NTG, spontaneous nociceptive behaviour was observed using a transparent acrylic apparatus $\left(45 \times 45 \times 35 \mathrm{~cm}^{3}\right)$. A camera was placed $1 \mathrm{~m}$ in front of the apparatus, and the behaviour of the rats was video-recorded for $20 \mathrm{~min}$. The rats' behaviour was analysed automatically using the rat behaviour recognition module of EthoVision XT 12.0 software (Noldus Information Technology, Leesburg, VA, United States).

Rat behaviour information and analysis were referenced from the descriptions in previous studies $[19,20]$. In brief, rat behaviour was categorized into five types: exploratory behaviour (including rearing up and sniffing), locomotor 


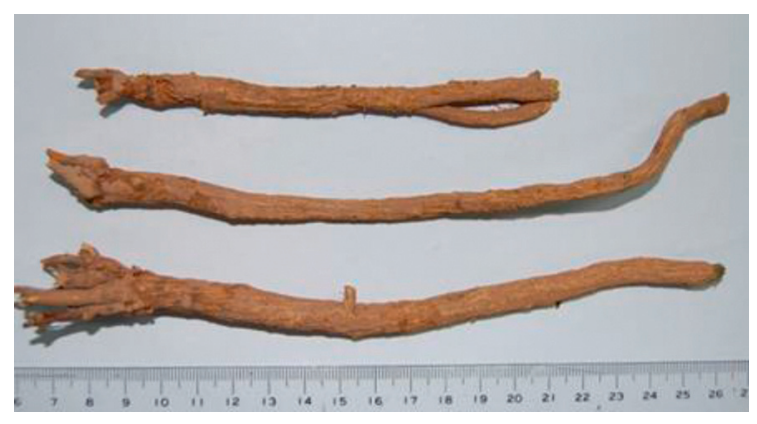

(a)

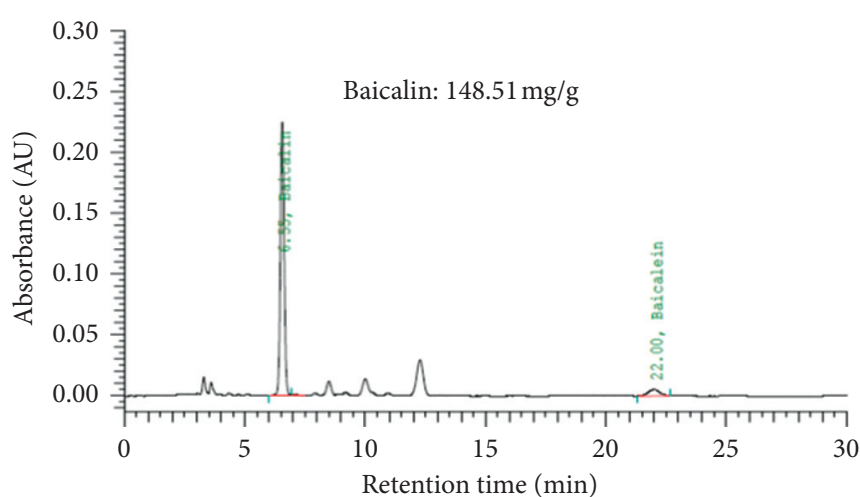

(b)

Figure 1: (a) Morphology of Scutellaria baicalensis (Huang Qin). (b) High-performance liquid chromatography (HPLC) fingerprint of Scutellaria baicalensis extract subtle granules.

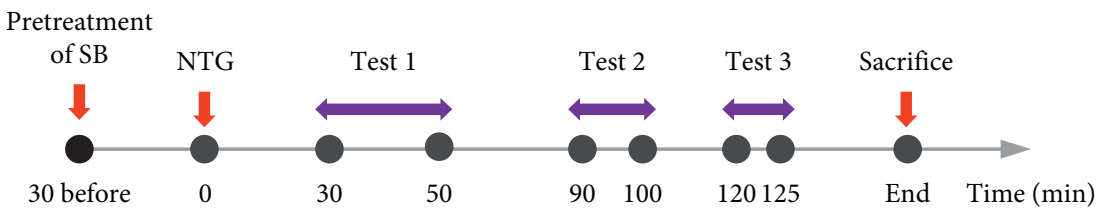

FIGURE 2: Experimental procedure. Pretreatment with Scutellaria baicalensis (SB): oral administration of Scutellaria baicalensis (SB) 30 min prior to i.p. injection with NTG; NTG: nitroglycerin $(10 \mathrm{mg} / \mathrm{kg})$ i.p. injection; test 1: rat behaviour recognition module test video recordings; test 2: light/dark box test video recordings; test 3: rough/smooth surface apparatus test video recordings.

behaviour (including walking and jumping), freezing behaviour (including twitching), resting behaviour, and grooming behaviour.

For the aforementioned behaviours, the frequency of engaging in the behaviour was calculated.

2.5.2. Assessment of Light-Aversive Behaviour. Ninety minutes after i.p. injection of NTG, light-aversive behaviour was tested using a light/dark box. The light/dark box was made of two identical compartments $\left(30 \times 30 \times 22.5 \mathrm{~cm}^{3}\right)$, where the light chamber was placed under a bright environment without a cover, and the dark box was fully black with a lid. A small opening gate $\left(10 \mathrm{~cm} \times 10 \mathrm{~cm}^{3}\right)$ connected the two chambers, and the rats could freely move across the two chambers. At the start of the assessment, the rats were placed in the centre of the light chamber and allowed to freely move across the two chambers for $10 \mathrm{~min}$. The test was video-taped and analysed using EthoVision XT 12.0. The parameter of total time spent in the light chamber was further analysed.

2.5.3. Assessment of Spontaneous Tactile Allodynia. One hundred and twenty minutes after the i.p. injection of NTG, spontaneous tactile allodynia was tested using a rough/ smooth surface apparatus, which was modified from a previous research [21]. The apparatus consisted of a transparent acrylic box $\left(45 \times 45 \times 35 \mathrm{~cm}^{3}\right)$, in which the floor was divided into two identical arenas $\left(22.5 \times 45 \mathrm{~cm}^{3}\right.$ each). The left-side floor surface was covered by smooth sandpaper
(P1000 grit), and the right-side floor surface was covered by rough sandpaper (P40 grit). At the beginning of the test, the rats were placed in the centre of the apparatus and allowed to freely move across both arenas for $5 \mathrm{~min}$. The test was videotaped and analysed using EthoVision XT 12.0. The total time spent in the smooth surface arena and heat map plots of the mean locations of the groups were calculated.

All rats were sacrificed after the completion of all behavioural tests.

2.6. Statistical Analysis. All the data are shown as mean\pm SEM. Statistical significance between the control, NTG, placebo, and SB-1.0 groups was analysed using one-way ANOVA followed by a Tukey's post hoc test. A $p$ value $<0.05$ was considered statistically significant. GraphPad Prism 7.0 software (San Diego, CA, USA) was used for the statistical analysis.

\section{Results}

3.1. Clinical Applications of SB in Taiwan. To investigate the clinical application of SB by TCM doctors in Taiwan, we conducted a population-based analysis using LHID 2000, which comprises a random sample of one million participants from the NHIRD between 1997 and 2013. The top 10 most frequent clinical applications of SB between 1997 and 2013 in Taiwan are shown in Table 1. The use of SB to treat headache $(N=4849,2.51 \%)$ was the fourth most common clinical application. 
TABle 1: Top 10 clinical applications of Scutellaria baicalensis by traditional Chinese medicine doctors from 1997 to 2013 in Taiwan.

\begin{tabular}{|c|c|c|c|c|}
\hline Ranking & ICD-9-CM & Indications & Usage number $(N)$ & Percentage (\%) \\
\hline 1 & 460 & Acute nasopharyngitis (common cold) & 25828 & 13.37 \\
\hline 2 & 786.2 & Cough & 15476 & 8.01 \\
\hline 3 & 477.9 & Allergic rhinitis cause unspecified & 4869 & 2.52 \\
\hline 4 & 784.0 & Headache & 4849 & 2.51 \\
\hline 5 & 780.59 & Other sleep disturbances & 2655 & 1.37 \\
\hline 6 & 490 & Bronchitis, not specified as acute or chronic & 2483 & 1.29 \\
\hline 7 & 472.0 & Chronic rhinitis & 2472 & 1.28 \\
\hline 8 & 780.50 & Sleep disturbances, unspecified & 2445 & 1.27 \\
\hline 9 & 536.9 & Unspecified functional disorder of stomach & 2339 & 1.21 \\
\hline 10 & 536.8 & Dyspepsia and other specified disorders of function of stomach & 2255 & 1.17 \\
\hline
\end{tabular}

ICD-9-CM: International Classification of Diseases, Ninth Revision, Clinical Modification.

\subsection{Effect of Pretreatment with SB on NTG-Induced Spontaneous Nociceptive Behaviour in Rats}

3.2.1. Exploratory Behaviour including Rearing Up and Sniffing. Notably, the rats in the NTG group engaged less in rearing up behaviour than those in the control group did $(p<0.001$; Figure 3(a) (A)). Pretreatment with $1.0 \mathrm{~g} / \mathrm{kg} \mathrm{SB}$ $(p<0.01$; Figure 3(a) (A)), but not placebo $(p>0.05$; Figure $3(\mathrm{a})(\mathrm{A})$ ), resulted in relatively more frequent rearing up behaviour compared to that in the NTG group.

Similar to the above outcome, the rats in the NTG group did not engage in sniffing behaviour as frequently as those in the control group did $(p<0.01$; Figure 3(a) (B)). Pretreatment with $1.0 \mathrm{~g} / \mathrm{kg} \mathrm{SB}(p<0.05$; Figure 3(a) (B)), but not placebo $(p>0.05$; Figure $3(\mathrm{a})(\mathrm{B}))$, resulted in a more frequent sniffing behaviour compared to that of the NTG group.

3.2.2. Locomotor Behaviour including Walking and Jumping. The rats in the NTG group engaged in walking behaviour less frequently than the control group $(p<0.001$; Figure 3 (b) (A)). Pretreatment with $1.0 \mathrm{~g} / \mathrm{kg} \mathrm{SB}(p<0.001$; Figure 3(b) $(\mathrm{A}))$, but not with placebo $(p>0.05$; Figure 3(b) (A)), resulted in a higher frequency of walking behaviour in comparison with that of the NTG group.

No significant differences were noted between the control, NTG, placebo, and SB-1.0 g/kg groups (all $p>0.05$; Figure $3(\mathrm{~b})(\mathrm{B}))$ in the analysis of the frequency of jumping behaviour.

3.2.3. Freezing Behaviour including Twitching. The rats in the NTG group did not show significantly different twitching behaviour from that of rats in the control group $(p>0.05$; Figure 3(c)). Pretreatment with $1.0 \mathrm{~g} / \mathrm{kg}$ SB led to more frequent twitching behaviour compared to that in the NTG group $(p<0.05$; Figure 3(c)). However, pretreatment with placebo resulted in a similar frequency of twitching behaviour as that in the NTG group $(p>0.05$; Figure $3(\mathrm{c}))$.

3.2.4. Resting Behaviour. The prevalence of resting behaviour was higher in the rats in the NTG group than that in the control group $(p<0.001$, Figure $3(d))$. Pretreatment with $1.0 \mathrm{~g} / \mathrm{kg} \mathrm{SB}(p<0.01$; Figure $3(\mathrm{~d}))$, but not placebo $(p>0.05$;
Figure 3(d)), resulted in lower engagement in resting behaviour compared to that in the NTG group.

3.2.5. Grooming Behaviour. The rats in the NTG group exhibited grooming behaviour more frequently than the control group did, and the difference was significant $(p<0.01$; Figure 3(e)). Pretreatment with $1.0 \mathrm{~g} / \mathrm{kg} \mathrm{SB}$ $(p<0.01$; Figure $3(\mathrm{e}))$, but not with placebo $(p>0.05$; Figure $3(\mathrm{e}))$, resulted in a decrease in the frequency of this behaviour.

3.2.6. Effect of Pretreatment with SB on NTG-Induced Light Aversion in Rats. The rats in the NTG group spent significantly less time in the light chamber compared to that spent by the control group $(p<0.05$; Figure 4$)$. Rats pretreated with $1.0 \mathrm{~g} / \mathrm{kg} \mathrm{SB}$ showed a difference in behaviour that approached marginal significance $(p=0.054$; Figure 4$)$, which was not observed with placebo pretreatment ( $p=0.977$; Figure 4 ), and spent a longer time in the light chamber than rats in the NTG group did.

3.2.7. Effect of Pretreatment with SB on NTG-Induced Spontaneous Tactile Allodynia Behaviour in Rats. The rats in the NTG group spent considerably longer time on the smooth surface than those in the control group did $(p<0.001$; Figure 5(a)). The time spent engaging in this behaviour was decreased by pretreatment with $1.0 \mathrm{~g} / \mathrm{kg} \mathrm{SB}$ $(p<0.05$; Figure 5(a)). Group mean location heat maps demonstrated that rats in the NTG group had a higher preference for the arena border and corners and spent more time on the smooth surfaces than those in the control group did (Figure 5(b)). This behaviour was decreased by pretreatment with $1.0 \mathrm{~g} / \mathrm{kg} \mathrm{SB}$ (Figure 5(b)).

\section{Discussion}

Migraine is considered a headache disorder, which involves the neural and vascular components of nociceptive transmission, and is associated with multiple pathophysiology, such as inflammation, and impaired functioning of neurotransmitters, ion channels, immune system, mitochondrial function, and oxidative stress factors [22]. Although Western medicine has rapidly developed treatments for 

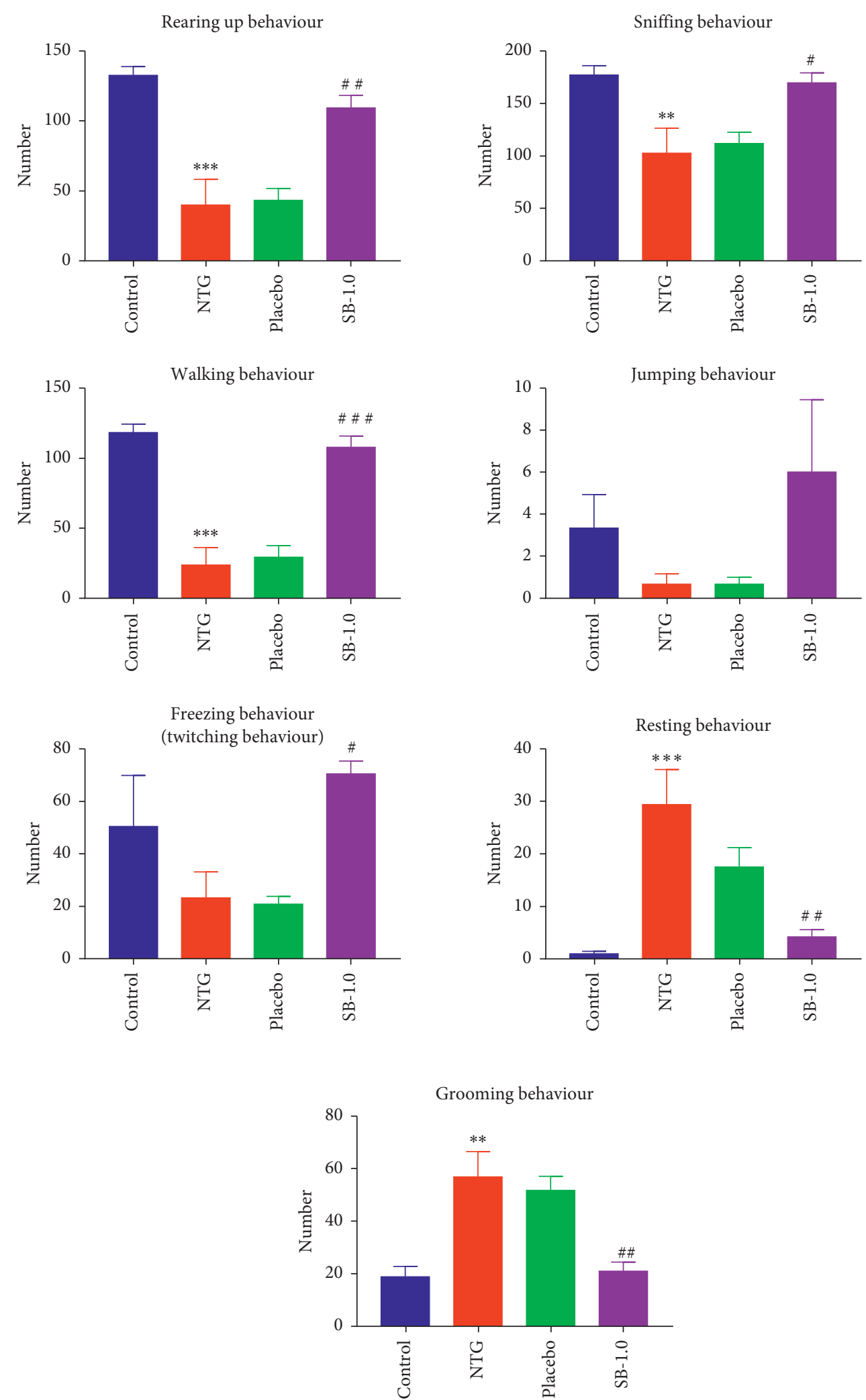

FIGURE 3: Effects of pretreatment with Scutellaria baicalensis (SB) on spontaneous nociceptive behaviour in a nitroglycerin-induced migraine rat model: (a) (A) the frequency of rearing up behaviour (exploratory behaviour); (B) the frequency of sniffing behaviour (exploratory behaviour). (b) (A) The frequency of walking behaviour (locomotor behaviour); (B) the frequency of jumping behaviour (locomotor behaviour). (c) The frequency of twitching behaviour (freezing behaviour). (d) The frequency of having an immobile posture or sleeping (resting behaviour). (e) The frequency of grooming the face or body (grooming behaviour). Frequency: the numbers/20 min; control: control group; NTG: NTG group; placebo: placebo group; SB-1.0: SB-1.0 group; data are presented as mean \pm SEM. $* p<0.05,{ }^{* *} p<0.01$, and ${ }^{* * *} p<0.001$ for the NTG group versus the control group; $\# p<0.05,{ }^{\# \#} p<0.01$, and ${ }^{\# \#} p<0.001$ versus the NTG group. 


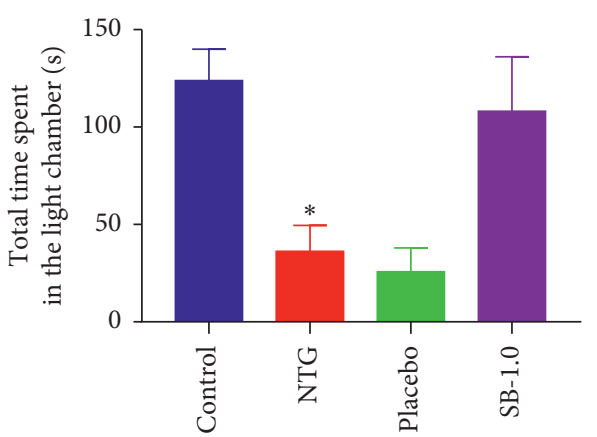

FIGURE 4: Effects of pretreatment with Scutellaria baicalensis (SB) on total time spent in the light chamber (s/10 min) of light-aversive behaviour in a nitroglycerin-induced migraine rat model. Control: control group; NTG: NTG group; placebo: placebo group; SB-1.0: SB-1.0 group; data are presented as mean \pm SEM. $* p<0.05, * * p<0.01$, and $* * * p<0.001$ for the NTG group versus the control group; $\# p<0.05,{ }^{\# \#} p<0.01$, and ${ }^{\# \# \#} p<0.001$ versus the NTG group.

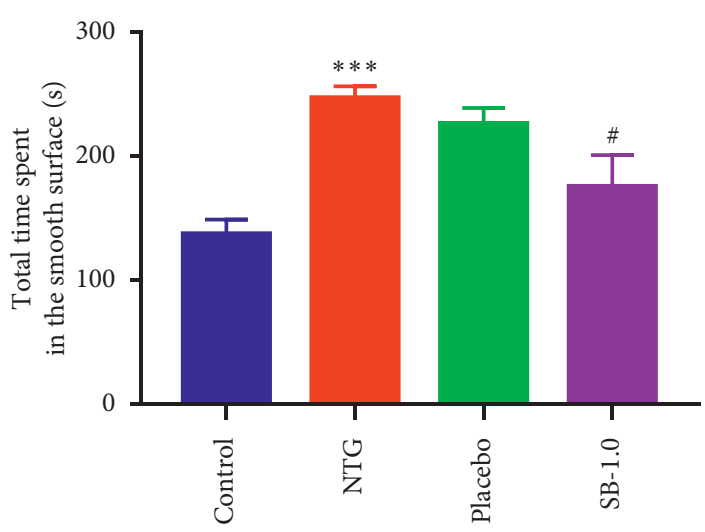

(a)

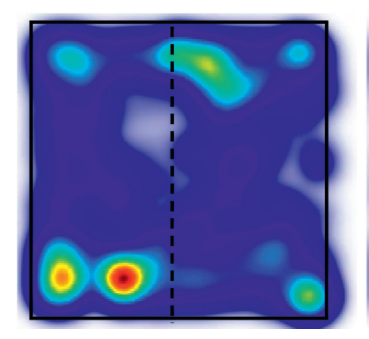

Smooth rough

Control

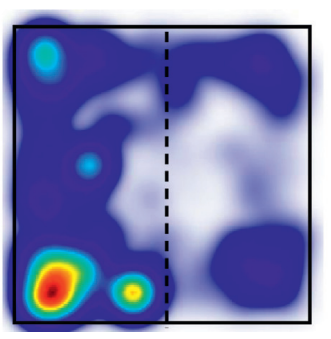

Smooth rough NTG

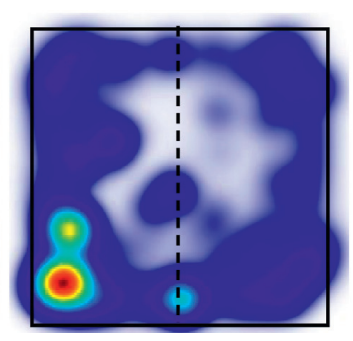

Smooth rough

Placebo

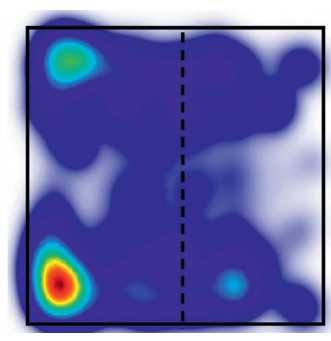

Smooth rough SB-1.0

(b)

FIGURE 5: Effects of pretreatment with Scutellaria baicalensis (SB) on spontaneous tactile allodynia behaviour in an NTG-induced migraine rat model. (a) Total time spent engaging in spontaneous tactile allodynia behaviour on the smooth surface ( $/ 5$ min); (b) group mean heat map plot (the left side is the smooth surface arena). Control: control group; NTG: NTG group; placebo: placebo group; SB-1.0: SB-1.0 group; smooth: smooth surface; rough: rough surface. Data are presented as mean \pm SEM. $* p<0.05, * * p<0.01$, and $* * * p<0.001$ for the NTG group versus the control group; $\# p<0.05,{ }^{\# \#} p<0.01$, and ${ }^{\# \# \#} p<0.001$ versus the NTG group.

migraine, some patients who face adverse effects of these medications seek alternative therapies for migraine prophylaxis and treatment [23]. Based on TCM theories and records in Chinese historical book, SB is considered an excellent anti-inflammatory and analgesic drug in the experience of traditional Chinese doctors; its use can be traced to the book "Shen Nong Ben Cao Jing" written in the Han Dynasty [8]. Moreover, SB is not only widely used in China but is also used as a medicinal plant in many countries around the world. For example, SB is a traditional plant medicine applied to wounds and insect bites in Nepal [8]. The chemical components, such as flavonoids, diterpenes, polyphenols, and amino acids, of SB [24] have several important biological activities, including anti-inflammatory, antitumor, antioxidant, antibacterial, and antiviral effects [25]. In addition, a recent study showed that SB has potential 
therapeutic effects on coronavirus disease 2019 [26]. Moreover, baicalin and baicalein, which are the major bioactive compounds in SB, have demonstrated an important role in inhibiting the production of inflammatory cytokines [25, 27], NF- $\kappa \mathrm{B}$ signalling [28, 29], and $\mathrm{c}$-fos expression [30] in multiple experimental models. These mechanisms are crucial in the pathophysiological processes of migraine. Hence, we assumed that SB could be potentially effective in migraine treatment.

However, to date, there has been no large-scale exploration of the actual clinical use of SB, including the distribution and frequency of use. In Taiwan, Chinese medicine is a popular medicine system for people. We also have a robust healthcare system with complete database records. Hence, we conducted a retrospective nationwide investigation of the prescription practices of TCM doctors to understand the actual clinical application of SB. Our findings revealed that SB was recommended for a variety of diseases, mainly respiratory inflammatory disorders, headache, sleep impairments, and gastrointestinal discomfort, in Taiwan. These findings are similar to those of a previous study, which showed that SB is commonly used for and has excellent therapeutic effects in sleep disturbance, hepatitis, diarrhoea, vomiting, haemorrhage, hypertension, and respiratory infection [8]. We found that SB could be used in varied diseases and is not limited to specific conditions; hence, the percentage of top ten diseases where SB was used was not particularly high. Although only $2.51 \%$ of the indications (the fourth most common indication) of SB in Taiwan was for the treatment of headaches, including migraine, surprisingly, it was not included in the clinical or basic applications of SB in previous scientific reports. It should be noted that our clinical investigation of the indications for SB usage is very credible because we used a nationwide random sample from the NHIRD, which had minimal selection bias owing to the very high rate of insured individuals and the fact that all Chinese herbal products were prescribed by welltrained and qualified TCM practitioners in Taiwan. Hence, we further designed an animal experiment using the widely accepted NTG-induced migraine model to prove the effectiveness of SB.

In the present study, we assessed various migraine-like behaviours of NTG-induced migraine rat models by using recorded videos that were analysed by reliable automated software [31, 32]; therefore, the results of the present study could be considered more credible and more objective than manually scored behaviours.

Migraine diagnosis is based on clinical self-reported pain and attendant symptoms. Although animals are not verbal, recent studies have considered NTG-induced animal models as clinically relevant due to the translational ability of behavioural endpoints [12, 21]. Our previous studies showed that NTG-induced nociceptive behaviours in rats with migraine headache included reduced exploratory and locomotor behaviours and increased resting behaviour $[33,34]$. The aforementioned nociceptive behaviours in rodents mimicked those experienced by people with migraine, who generally present with reduced involvement in routine physical activity and decreased interest in exploring new surroundings or objects during an acute migraine attack episode $[34,35]$. Therefore, we could use the parameters of the rat model to test antianalgesic drug potency. The results of the present study demonstrated that pretreatment with $1.0 \mathrm{~g} / \mathrm{kg} \mathrm{SB}$ could increase locomotor activity and decrease resting behaviour in NTGinduced migraine rats, suggesting that pretreatment with SB could alleviate migraine pain in humans.

Photophobia is one of the important symptoms of migraine [36]. Previous reports have shown that light aversion behaviour, tested using the light/dark box, in NTG-induced migraine rodents effectively mimics the phenomenon of photophobia [35, 37]. Our results indicated that pretreatment with $1.0 \mathrm{~g} / \mathrm{kg} \mathrm{SB}$ resulted in decreased light aversion, which approached marginal significance, implying that SB might alleviate photophobia in an individual who experiences migraine.

Cutaneous allodynia is a common symptom seen in migraineurs; it indicates cranial hypersensitivity and appears to be a predictor of migraine chronification [38, 39]. Recently, a rough/smooth apparatus test was used to evaluate spontaneous tactile allodynia in an NTG-induced migraine rodent model [21]. Our results showed that pretreatment with $1.0 \mathrm{~g} / \mathrm{kg} \mathrm{SB}$ decreased the total time spent on the smooth surface in the rough/smooth test, indicating that SB can decrease tactile allodynia in NTG-induced migraine rat models, which suggests that SB can be helpful for the clinical treatment of cutaneous allodynia in migraineurs.

There are still some limitations to the present study: (1) the animal experiment lacked a group treated with a medicine, such as ibuprofen, to compare the efficacy of SB and conventional Western medicine. In addition, a dosedependent relationship of the bioactive agents of SB, such as baicalin, was not reported in the current animal experiment; (2) the regulatory mechanisms of SB in migraine rats were not explored, and further validation, such as by biomarker determination (NO, CGRP level) and immunohistochemistry assay, should be conducted to investigate neuronal activity in the future; and (3) although the results of the present study explain how SB was helpful for the treatment of NTG-induced migraine in rats, a welldesigned clinical trial is required to evaluate the efficacy of $\mathrm{SB}$ in humans.

\section{Conclusions}

The results of the present study indicated that pretreatment with SB increased the frequency of rearing up and sniffing in exploratory behaviour and increased the frequency of walking in locomotor behaviour in NTG-induced migraine models. NTG-induced migraine rats pretreated with SB also had lower frequencies of both resting behaviour and grooming behaviour. These phenomena indicate that SB could be helpful in decreasing the migraine pain level. Additionally, NTG-induced migraine rats pretreated with $\mathrm{SB}$ spent less time on the smooth surface compared to the time spent by the rats that did not receive SB pretreatment, which indicates that SB could be helpful in decreasing cutaneous allodynia in migraineurs. These results support the 
application of SB for the treatment of headache, as is a common practice in Taiwan.

$\begin{array}{ll}\text { Abbreviations } \\ \text { i.p.: } & \text { Intraperitoneal injection } \\ \text { ICD-9- } & \text { International Classification of Diseases, Ninth } \\ \text { CM: } & \text { Revision, Clinical Modification } \\ \text { NHIRD: } & \text { National Health Insurance Research Database } \\ \text { NTG: } & \text { Nitroglycerin } \\ \text { SB: } & \text { Scutellaria baicalensis } \\ \text { TCM: } & \text { Traditional Chinese medicine. }\end{array}$

\section{Data Availability}

The datasets used and analysed during the current study are available from the corresponding author upon reasonable request.

\section{Ethical Approval}

1. Human data: this study was approved by the Institutional Review Board of China Medical University in central Taiwan (CMUH-104-REC2-115-R3). 2. Animals: animal use was approved by the Institutional Animal Care and Use Committee of Show Chwan Memorial Hospital (no. 106021) and followed the Guide for the Use of Laboratory Animals (National Academy Press).

\section{Conflicts of Interest}

The authors declare that they have no conflicts of interest.

\section{Authors' Contributions}

C-C Liao and K-R Liao performed the animal experiment and wrote the manuscript, C-L Lin analysed the data from the NHIRD in Taiwan, and J-M Li designed the protocol and revised the manuscript. All authors read and approved the final manuscript. Chung-Chih Liao and Ke-Ru Liao contributed equally to this work.

\section{Acknowledgments}

This study was supported by a grant from Show Chwan Memorial Hospital (RD106087) and also supported in part by the Taiwan Ministry of Health and Welfare Clinical Trial Center (MOHW109-TDU-B-212-114004), MOST Clinical Trial Consortium for Stroke (MOST 108-2321-B-039-003-), and Tseng-Lien Lin Foundation, Taichung, Taiwan.

\section{References}

[1] R. Burstein, R. Noseda, and D. Borsook, "Migraine: multiple processes, complex pathophysiology," Journal of Neuroscience, vol. 35, no. 17, pp. 6619-6629, 2015.

[2] Y. Woldeamanuel and R. Cowan, "Worldwide migraine epidemiology: systematic review and meta-analysis of 302 community-based studies involving 6,216,995 participants (P6.100)," Neurology, vol. 86, no. 16, Article ID P6.100, 2016.
[3] R. C. Burch, D. C. Buse, and R. B. Lipton, "Migraine: epidemiology, burden, and comorbidity," Neurologic Clinics, vol. 37, no. 4, pp. 631-649, 2019.

[4] C.-T. Liu, B.-Y. Wu, Y.-C. Hung et al., "Decreased risk of dementia in migraine patients with traditional Chinese medicine use: a population-based cohort study," Oncotarget, vol. 8, no. 45, pp. 79680-79692, 2017.

[5] D. Millstine, C. Y. Chen, and B. Bauer, "Complementary and integrative medicine in the management of headache," $B M J$, vol. 357, Article ID j1805, 2017.

[6] Y. Zhao, M. Martins-Oliveira, S. Akerman, and P. J. Goadsby, "Comparative effects of traditional Chinese and western migraine medicines in an animal model of nociceptive trigeminovascular activation," Cephalalgia, vol. 38, no. 7, pp. 1215-1224, 2018.

[7] S. Yu, Y. Ran, W. Xiao et al., "Treatment of migraines with Tianshu capsule: a multi-center, double-blind, randomized, placebo-controlled clinical trial," BMC Complementary and Alternative Medicine, vol. 19, no. 1, p. 370, 2019.

[8] T. Zhao, H. Tang, L. Xie et al., "Scutellaria baicalensis Georgi. (Lamiaceae): a review of its traditional uses, botany, phytochemistry, pharmacology and toxicology," Journal of Pharmacy and Pharmacology, vol. 71, no. 9, pp. 1353-1369, 2019.

[9] S. Hu, Y. Chen, Z. F. Wang et al., "The analgesic and antineuroinflammatory effect of baicalein in cancer-induced bone pain," Evidence-Based Complementary and Alternative Medicine, vol. 2015, Article ID 973524, 8 pages, 2015.

[10] S.-B. Yoon, Y.-J. Lee, S. K. Park et al., “Anti-inflammatory effects of Scutellaria baicalensis water extract on LPS-activated RAW 264.7 macrophages," Journal of Ethnopharmacology, vol. 125, no. 2, pp. 286-290, 2009.

[11] C. Waeber and M. A. Moskowitz, "Migraine as an inflammatory disorder," Neurology, vol. 64, no. 10, pp. S9-S15, 2005.

[12] K. J. Sufka, S. M. Staszko, A. P. Johnson, M. E. Davis, R. E. Davis, and T. A. Smitherman, "Clinically relevant behavioral endpoints in a recurrent nitroglycerin migraine model in rats," Journal of Headache and Pain, vol. 17, p. 40, 2016.

[13] C. Tassorelli, R. Greco, D. Wang, M. Sandrini, G. Sandrini, and G. Nappi, "Nitroglycerin induces hyperalgesia in rats-a time-course study," European Journal of Pharmacology, vol. 464, no. 2-3, pp. 159-162, 2003.

[14] C. Tassorelli and S. A. Joseph, "Systemic nitroglycerin induces Fos immunoreactivity in brainstem and forebrain structures of the rat," Brain Research, vol. 682, no. 1-2, pp. 167-181, 1995.

[15] F. Farajdokht, S. Babri, P. Karimi, and G. Mohaddes, "Ghrelin attenuates hyperalgesia and light aversion-induced by nitroglycerin in male rats," Neuroscience Letters, vol. 630, pp. 30-37, 2016.

[16] C. H. Chen, C. L. Lin, and C. H. Kao, "Gallbladder stone disease is associated with an increased risk of migraines," Journal of Clinical Medicine, vol. 7, no. 11, p. 455, 2018.

[17] Y. J. Wang, C. C. Liao, H. J. Chen, C. L. Hsieh, and T. C. Li, "The effectiveness of traditional Chinese medicine in treating patients with leukemia," Evidence-Based Complementary and Alternative Medicine, vol. 2016, Article ID 8394850, 12 pages, 2016.

[18] A. Nair and S. Jacob, "A simple practice guide for dose conversion between animals and human," Journal of Basic and Clinical Pharmacy, vol. 7, no. 2, pp. 27-31, 2016.

[19] A. Melo-Carrillo and A. Lopez-Avila, "A chronic animal model of migraine, induced by repeated meningeal nociception, characterized by a behavioral and pharmacological approach," Cephalalgia, vol. 33, no. 13, pp. 1096-1105, 2013. 
[20] B. Vos, A. Strassman, and R. Maciewicz, "Behavioral evidence of trigeminal neuropathic pain following chronic constriction injury to the rat's infraorbital nerve," The Journal of Neuroscience, vol. 14, no. 5, pp. 2708-2723, 1994.

[21] H. M. Harris, J. M. Carpenter, J. R. Black, T. A. Smitherman, and K. J. Sufka, "The effects of repeated nitroglycerin administrations in rats; modeling migraine-related endpoints and chronification," Journal of Neuroscience Methods, vol. 284, pp. 63-70, 2017.

[22] C. Demartini, R. Greco, A. M. Zanaboni et al., "Nitroglycerin as a comparative experimental model of migraine pain: from animal to human and back," Progress in Neurobiology, vol. 177, pp. 15-32, 2019.

[23] C. A. Whyte and S. J. Tepper, "Adverse effects of medications commonly used in the treatment of migraine," Expert Review of Neurotherapeutics, vol. 9, no. 9, pp. 1379-1391, 2009.

[24] B. Dinda, S. Dinda, S. DasSharma, R. Banik, A. Chakraborty, and M. Dinda, "Therapeutic potentials of baicalin and its aglycone, baicalein against inflammatory disorders," European Journal of Medicinal Chemistry, vol. 131, pp. 68-80, 2017.

[25] H. Liao, J. Ye, L. Gao, and Y. Liu, "The main bioactive compounds of Scutellaria baicalensis Georgi. for alleviation of inflammatory cytokines: a comprehensive review," Biomedicine \& Pharmacotherapy, vol. 133, Article ID 110917, 2020.

[26] J. W. Song, J. Y. Long, L. Xie et al., "Applications, phytochemistry, pharmacological effects, pharmacokinetics, toxicity of Scutellaria baicalensis Georgi. and its probably potential therapeutic effects on COVID-19: a review," Chinese Medicine, vol. 15, p. 102, 2020

[27] J. Zhang, C. Teng, C. Li, and W. He, "Deliver anti-inflammatory drug baicalein to macrophages by using a crystallization strategy," Frontiers in Chemistry, vol. 8, p. 787, 2020.

[28] M. Zou, L. Yang, L. Niu et al., "Baicalin ameliorates Mycoplasma gallisepticum-induced lung inflammation in chicken by inhibiting TLR6-mediated NF- $\kappa \mathrm{B}$ signalling," British Poultry Science, pp. 1-12, 2020.

[29] Y. Ji, J. Han, N. Lee et al., "Neuroprotective effects of baicalein, wogonin, and oroxylin A on amyloid beta-induced toxicity via NF- $\kappa$ B/MAPK pathway modulation," Molecules, vol. 25, no. 21, Article ID 5087, 2020.

[30] K.-C. Kim, S.-S. Kang, J.-S. Lee, D.-H. Park, and J.-W. Hyun, "Baicalein attenuates oxidative stress-induced expression of matrix metalloproteinase-1 by regulating the ERK/JNK/AP-1 pathway in human keratinocytes," Biomolecules and Therapeutics, vol. 20, no. 1, pp. 57-61, 2012.

[31] E. A. van Dam, J. E. van der Harst, C. J. F. ter Braak, R. A. J. Tegelenbosch, B. M. Spruijt, and L. P. J. J. Noldus, “An automated system for the recognition of various specific rat behaviours," Journal of Neuroscience Methods, vol. 218, no. 2, pp. 214-224, 2013.

[32] F. C. P. Simão, F. Martínez-Jerónimo, V. Blasco et al., "Using a new high-throughput video-tracking platform to assess behavioural changes in Daphnia magna exposed to neuroactive drugs," Science of the Total Environment, vol. 662, pp. 160-167, 2019.

[33] C.-C. Liao, J.-M. Li, C.-H. Chen, C.-L. Lin, and C.-L. Hsieh, "Effect of Paeonia lactiflora, a traditional Chinese herb, on migraines based on clinical application and animal behavior analyses," Biomedicine \& Pharmacotherapy, vol. 118, Article ID 109276, 2019.

[34] C. C. Liao, J. M. Li, and C. L. Hsieh, "Auricular electrical stimulation alleviates headache through CGRP/COX-2/ TRPV1/TRPA1 signaling pathways in a nitroglycerin-induced migraine rat model," Evidence-Based Complementary and
Alternative Medicine, vol. 2019, Article ID 2413919, 10 pages, 2019.

[35] D. Vuralli, A. S. Wattiez, A. F. Russo, and H. Bolay, "Behavioral and cognitive animal models in headache research," The Journal of Headache and Pain, vol. 20, no. 1, p. 11, 2019.

[36] K. B. Digre and K. C. Brennan, "Shedding light on photophobia," Journal of Neuro-Ophthalmology, vol. 32, no. 1, pp. 68-81, 2012.

[37] F. Farajdokht, S. Babri, P. Karimi, M. R. Alipour, R. Bughchechi, and G. Mohaddes, "Chronic ghrelin treatment reduced photophobia and anxiety-like behaviors in nitroglycerin- induced migraine: role of pituitary adenylate cyclase-activating polypeptide," European Journal of Neuroscience, vol. 45, no. 6, pp. 763-772, 2017.

[38] S. Akerman, M. Romero-Reyes, N. Karsan et al., "Therapeutic targeting of nitroglycerin-mediated trigeminovascular neuronal hypersensitivity predicts clinical outcomes of migraine abortives," Pain, 2020.

[39] M. A. Louter, J. E. Bosker, W. P. van Oosterhout et al., "Cutaneous allodynia as a predictor of migraine chronification,” Brain, vol. 136, no. 11, pp. 3489-3496, 2013. 